\title{
CONTROL SYSTEM RESPONSE OF DIFFERENT RESPIRATORY MODELS UNDER VENTILATORY STIMULI AND PATHOLOGIES
}

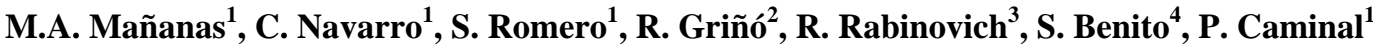 \\ ${ }^{1}$ Automatic Control Dept., Technical University of Catalonia (UPC), Barcelona, Spain \\ ${ }^{2}$ Institut d'Organització i Control de Sistemes Industrials, UPC, Barcelona, Spain \\ ${ }^{3}$ Servei de Pneumologia, Hospital Clínic i Provincial, IDIBAP, Barcelona, Spain \\ ${ }^{4}$ Unitat de Semicrítics, Hospital de les Santes Creus i Sant Pau, Barcelona, Spain
}

\begin{abstract}
The purpose of this work is to evaluate characteristics of a respiratory model, $R S 1$, in the presence of the following stimuli: exercise, hypercapnia and hypoxia. RS1 has a controller where the driving signal optimizes alveolar ventilation and respiratory frequency to minimize the respiratory work output. A comparative study of the steadystate and transient responses with other three models is performed by simulation. Alternative equations to calculate the optimum frequency are evaluated during exercise and a linear average of two of them is proposed and tested for a healthy subject and with restrictive/obstructive pathology. Additionally, the circulatory time delay in gas transport that produces periodic breathing is calculated. Copyright () 2002 IFAC
\end{abstract}

Keywords: Biomedical system, models, computer simulation.

\section{INTRODUCTION}

The respiratory control system is a nonlinear, multioutput, delayed-feedback dynamic system which is constantly being perturbed by physiologic and pathologic disturbances (Poon, 1995). Control of ventilation is automatic and normally involuntary in the presence of different stimuli: from a change in metabolic activity to a modification of external environment. This biological system is dedicated to a specific physiologic function: the exchange of $\mathrm{O}_{2}$ and $\mathrm{CO}_{2}$. Respiratory control has been traditionally modeled as a closed-loop system that adjusts the alveolar ventilation, $\dot{\mathrm{v}}_{\mathrm{A}}$, to the demands of the body so that the arterial $\mathrm{CO}_{2}$ pressure, $\mathrm{PaCO}_{2}$, and arterial $\mathrm{O}_{2}$ pressure, $\mathrm{PaO}_{2}$, remain practically constant. This is called homeostasis and occurs even during strenuous exercise or other types of respiratory stress (Guyton, 1986).

Many empirical and functional models have been proposed in the literature to describe various aspects of the respiratory system. Some of them correspond to a complete description of the closed-loop system and assume that it is structured in different intermediate processes. Every approach in the model implies a separate law of the multi-tasking controller. However, these control laws must be related each other, because they are regulated by the same neural network which composes the respiratory controller. Three of these models were studied in a previous work (Mañanas et al., 2000) because of the variety in modeling and control laws (Poon, 1987; Khoo et al., 1982, 1991).

Another much more complex model with more variables of physiological interest has been chosen in this work (Fincham and Tehrani, 1983). It has been compared with the previous models to analyze their responses in different situations of ventilation control: moderate exercise, hypercapnia and hypoxia. Pulmonary diseases have also been studied to evaluate the utility of the models in the analysis of pathologies. Static and dynamic ventilatory responses have been analyzed by means of simulation. The software used in this work is Simulink v3.0 from MATLAB on PCWIN. 


\section{METHODS}

\subsection{Respiratory models}

The first analyzed model is (Poon, 1987) with chemical and neuro-mechanical feedback (see Fig.1). The expired ventilation, $\overline{\mathrm{V}}_{\mathrm{E}}$, proceeds from an optimization approach to minimize chemical and mechanical works in ventilation maintaining the homeostasis. Although it is a static model, the chemical plant is changed by the dynamic equations that appear in (Poon, 1995).

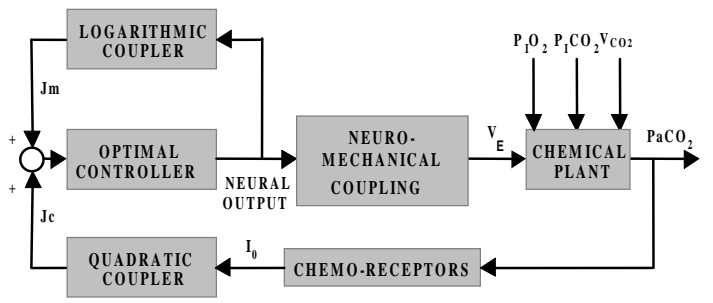

Fig. 1. Block diagram of the respiratory control system from (Poon, 1987).

The second model corresponds to (Khoo et al., 1982) whose block diagram fits better to physiological system with peripheral and central components (see Fig. 2). Both chemoreceptors, neural activity and respiratory muscle driving pressure are integrated in the peripheral and central controllers whose mission is the homeostasis.

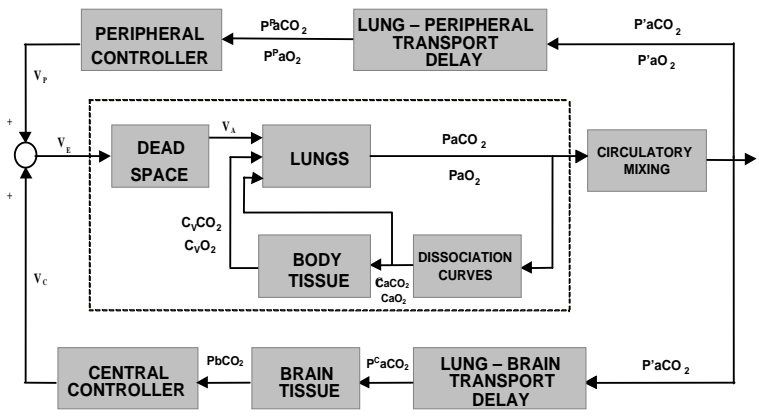

Fig. 2. Block diagram of the respiratory control system from (Khoo et al., 1982). Plant is framed inside the dashed box.

The third model (Khoo et al., 1991) is an extension of the second one (see Fig. 3). It presents the same chemical plant but the chemoreceptors do not act as proportional controllers but perform their real physiological function: get the $\mathrm{PaCO}_{2}$ and $\mathrm{PaO}_{2}$ and send this information to the controller located in the medulla. The model adds important variables in the respiratory pattern generation as tidal volume, $\mathrm{V}_{\mathrm{T}}$, and respiratory frequency, $\mathrm{f}$, although it is constant with the same duration in inspiration and expiration periods. In the chemical plant of last two models, there are more blocks and variables indicating physiological processes than the first one: gas concentrations in veins and arteries, $\left(\mathrm{C}_{\mathrm{v}} \mathrm{CO}_{2} / \mathrm{C}_{\mathrm{v}} \mathrm{O}_{2}\right.$ and $\mathrm{C}_{\mathrm{a}} \mathrm{CO}_{2} / \mathrm{C}_{\mathrm{a}} \mathrm{O}_{2}$, respectively), gas exchange in body tissue and brain, circulatory mixing and circulation time from tissues to chemoreceptors. Furthermore, there is a feedback of $\mathrm{PaO}_{2}$ by means of peripheral chemoreceptor whereas in (Poon, 1987) there is not.

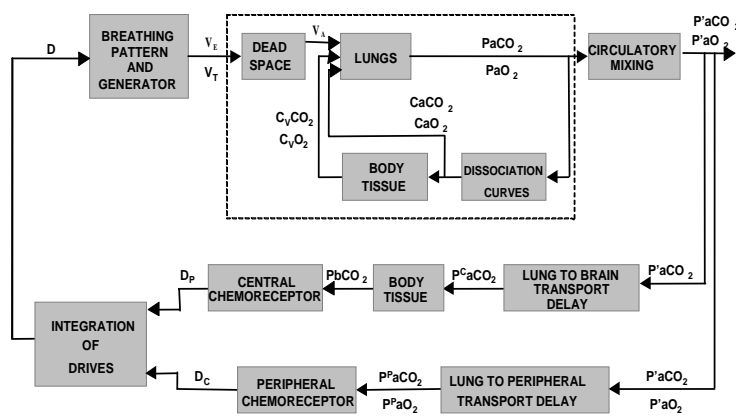

Fig. 3. Block diagram of the respiratory control system from (Khoo et al., 1991). Plant is framed inside the dashed box.

The last model (Fincham and Tehrani, 1983a) is the most complex. Besides including the biomedical variables mentioned before, it considers a controller whose parameters of respiratory pattern are calculated every cycle as it happens physiologically. Additionally, lung volume changes following a sinus during the respiratory cycle to simulate more properly inspiration and expiration intervals. In the other models, this evolution during the respiratory cycle is not considered. Therefore, driving signal, $\mathrm{dv} / \mathrm{dt}$, optimize $\dot{\mathrm{V}}_{\mathrm{A}}$ and $\mathrm{f}$ every breath to minimize the respiratory work output,

$$
\frac{d v}{d t}=\pi \cdot V_{A} \cdot \sin (2 \pi f t)
$$

Thus, Hering Breuer reflex (Guyton, 1986) is included permitting the controller to modify rate and amplitude of respiration at the end of every cycle. The mathematical model is completed in (Tehrani, 1993) where the author also changes some parameters to adjust its response to newborn infants (see Fig. 4).

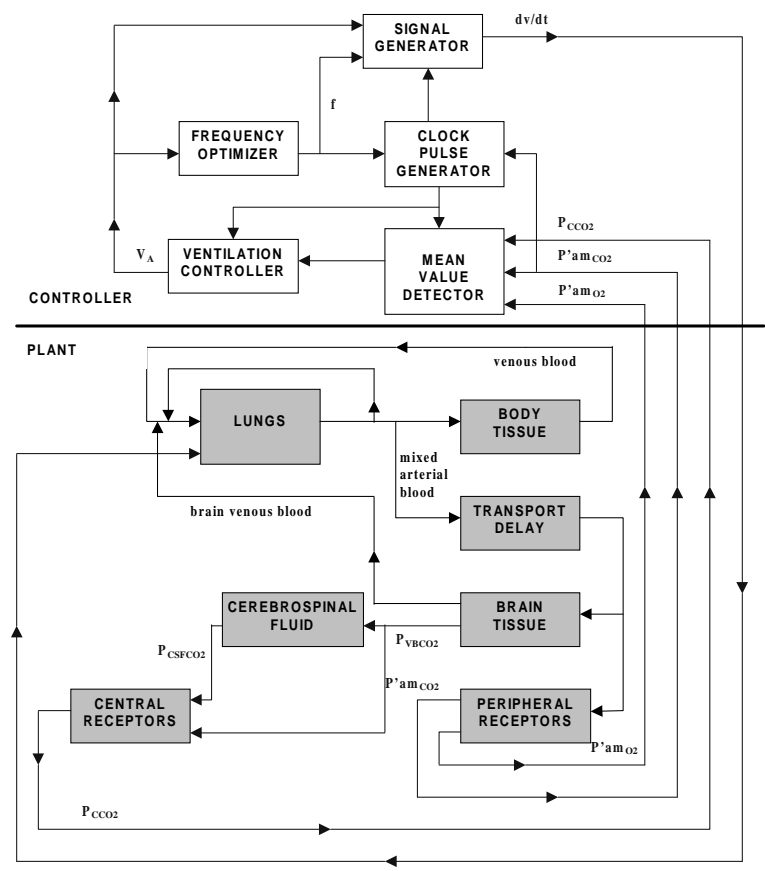

Fig. 4. Block diagram of the respiratory control system from (Tehrani, 1993). 
Interaction between cardiac output, $\mathrm{Q}$, and respiratory function $\left(\mathrm{PaCO}_{2}, \mathrm{PaO}_{2}\right.$, and metabolic rate) is considered in this last model. Mathematical expressions based on experimental data and composed by Chebyshev polynomials are described in (Fincham and Tehrani, 1983b).

\subsection{Stimuli}

Responses and characteristics of the four respiratory models in the presence of the following stimuli are evaluated: moderate exercise, hypercapnia and hypoxia. During exercise, $\mathrm{O}_{2}$ consumption and $\mathrm{CO}_{2}$ production, $\mathrm{VCO}_{2}$, can increase a lot. A value of $\mathrm{VCO}_{2}=0.2 \mathrm{l} / \mathrm{min}$ is considered at rest to a maximum value of $1 \mathrm{l} / \mathrm{min}$ with moderate exercise.

Hypoxia can result from disturbances of respiration or a reduction in the $\mathrm{O}_{2}$ pressure of inspired air as occurs at high altitudes. For the simulation, an input variable, $\mathrm{P}_{\mathrm{I}} \mathrm{O}_{2}$, is considered with values lower than 159 torr (21\% of atmospheric pressure), corresponding to normal conditions, until 98 torr (13\%) of maximum hypoxic condition. It is related with a decrease in $\mathrm{PaO}_{2}$. Hypoxia results are principally depressed mental activity and reduced work capacity of the muscles (Guyton, 1986).

Hypercapnia corresponds to a presence of $\mathrm{CO}_{2}$ in the inhaled gas or $\mathrm{CO}_{2}$ retention. An input variable, $\mathrm{P}_{\mathrm{I}} \mathrm{CO}_{2}$, is considered from 0 torr $(0 \%$ of atmospheric pressure) until 45 torr $(6 \%)$. It means excess $\mathrm{CO}_{2}$ in the body fluids. Dyspnea usually becomes very severe when $\mathrm{PaCO}_{2}$ rises above 40 or 55 torr. The person becomes lethargic and sometimes even semicomatose with levels from 80 to 100 torr (Guyton, 1986).

\section{RESULTS}

\subsection{Steady-state response}

In Fig. 5, final values of variables of interest $\left(V_{E}\right.$, $\mathrm{PaCO}_{2}, \mathrm{PaO}_{2}, \mathrm{~V}_{\mathrm{T}}$ and $\mathrm{f}$ ) as a function of different levels of exercise, hypoxia and hypercapnia are shown. Different line types are used to differentiate the steady-state response among the four respiratory models. A mean value of the differences between each couple of models with every stimulus or situation is shown in Table 1 by means of the Percentile Root-mean-square Difference (PRD):

$$
\operatorname{PRD}(\%)=\sqrt{\frac{\sum_{\mathrm{n}=1}^{\mathrm{N}}\left(\mathrm{x}_{1}(\mathrm{n})-\mathrm{x}_{2}(\mathrm{n})\right)^{2}}{\sum_{\mathrm{n}=1}^{\mathrm{N}} \mathrm{x}_{1}^{2}(\mathrm{n})}} 100
$$

where $x_{1}(n)$ and $x_{2}(n)$ corresponds to the respiratory variable from two models and $\mathrm{N}$ is the number of different levels of simulated stimulus.
Table 1 PRD (\%) between each couple of models in the respiratory variables: $\mathrm{V}_{\mathrm{E}}, \mathrm{PaCO}_{2}$ and $\mathrm{PaO}_{2}$ at

every stimulus or situation of exercise, hypercapnia and hypoxia. Models FT. P, K1 and K2 correspond to (Fincham and Tehrani, 1983a), (Poon, 1987), (Khoo et al, 1982, 1991) respectively.

\begin{tabular}{llccccc} 
& & FT-P & FT-K1 & FT-K2 & P-K1 & P-K2 \\
\hline \multirow{4}{*}{ EXERCISE } & $\mathrm{PaCO}_{2}$ & 5,60 & 11,27 & 12,33 & 14.69 & 15.46 \\
& $\mathrm{PaO}_{2}$ & 0,69 & 5,82 & 6,22 & 5.80 & 6.16 \\
\hline \multirow{2}{*}{ HYPER } & $\mathrm{V}_{\mathrm{E}}$ & 5,04 & 26,20 & 25,03 & 19.87 & 18.99 \\
CAPNIA & $\mathrm{PaCO}_{2}$ & 3,67 & 4,53 & 3,87 & 0.99 & 0.30 \\
& $\mathrm{PaO}_{2}$ & 0,79 & 0,41 & 0,76 & 0.41 & 0.12 \\
\hline \multirow{4}{*}{ HYPOXIA } & $\mathrm{PaCO}_{2}$ & 2,51 & 1,17 & 1,75 & 2.73 & 0.76 \\
& $\mathrm{VaO}_{2}$ & 2,11 & 1,73 & 2,01 & 1.65 & 0.56 \\
\hline
\end{tabular}

\subsection{Transient response}

The four dynamic models permit to evaluate the transient response of the variables of interest in a selected situation of exercise, hypercapnia or hypoxia. Whereas there are no overshoots in any response in (Poon, 1987), they appear in $\mathrm{PaO}_{2}$ in (Khoo et al., 1982, 1991) because of the existence of an exponential feedback of $\mathrm{PaO}_{2}$. An overshoot is also obtained in $\mathrm{PaO}_{2}$ and another smaller in $\mathrm{PaCO}_{2}$ in (Fincham and Tehrani, 1983a) because of the same reason. However, this overshoot is opposite to the response found in Khoo's models (see Fig. 6) and it is associated with the physiological transient response (Guyton, 1986).

Furthermore, a settling time, $\mathrm{t}_{\mathrm{s}},( \pm 5 \%$ of final value $)$ of several seconds is found in model (Poon, 1987) and a more realistic value of some minutes is obtained in the Khoo's models. For example, with a step from $0 \%$ to $3 \%$ in $\mathrm{P}_{\mathrm{I}} \mathrm{CO}_{2}, \mathrm{t}_{\mathrm{s}}$ for $\mathrm{PaCO}_{2}$ variable is $15 \mathrm{~s}, 525 \mathrm{~s}$ and $450 \mathrm{~s}$ in models (Poon, 1987), and (Khoo et al., 1987, 1991), respectively. Otherwise, $t_{\mathrm{s}}$ is too long, 45 minutes, in (Fincham and Tehrani, 1983a). This model dynamics is also very slow in hypoxia but properly in exercise where $t_{s}$ is around 2 minutes for the most important variables.

\subsection{Optimization of respiratory frequency}

Respiratory rate is calculated in the model (Fincham and Tehrani, 1983a) on a minimum respiratory work basis as found by Otis et al. (1950). Turbulence forces and inertia of the lungs are neglected considering all the work performed during inspiration interval. Lung's elastance, $\gamma$, airway resistance, $\lambda$, and sinusoidal flow waveform as given by equation (1) are considered in the respiratory frequency:

$$
f_{\text {Otis }}=\frac{-\gamma V_{D}+\sqrt{\left(\gamma V_{D}\right)^{2}+4 \gamma \lambda \pi^{2} V_{D} \dot{V}_{A}}}{2 \pi^{2} \lambda V_{D}}
$$



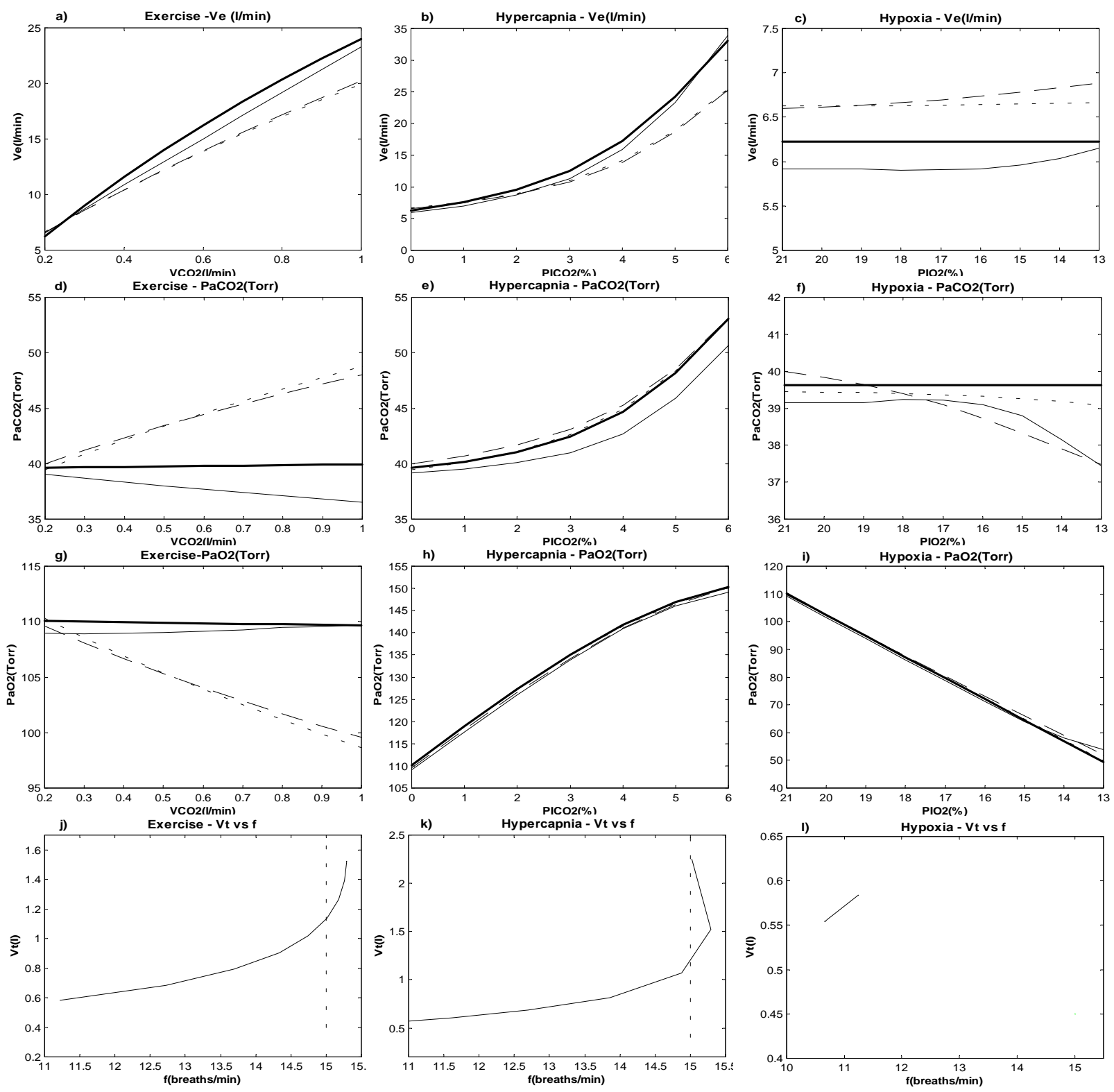

Fig. 5. Steady state values of $\mathrm{V}_{\mathrm{A}}$ (a), b), c)), $\left.\left.\mathrm{PaCO}_{2}(\mathrm{~d}), \mathrm{e}\right), \mathrm{f}\right)$ ) and $\left.\left.\mathrm{PaO}_{2}(\mathrm{~g}), \mathrm{h}\right), \mathrm{i}\right)$ ) as a function of $\dot{\mathrm{V}}_{\mathrm{CO}}$ in exercise (a), d), g)), $\mathrm{P}_{\mathrm{I}} \mathrm{CO}_{2}$ in hypercapnia (b), e), h)), and $\mathrm{P}_{\mathrm{I}} \mathrm{O}_{2}$ in hypoxia (c), f), i)). The curves $\mathrm{V}_{\mathrm{T}}$ vs. $\mathrm{f}$ are very useful for the physiologists and are calculated during exercise (j)), hypercapnia (k)) and hypoxia (1)). Different line types are used to each respiratory model: continuous (Fincham and Tehrani, 1983a), continuous bold (Poon, 1987), dashed (Khoo et al, 1982) and dotted (Khoo et al., 1991).

where $V_{D}$ is the dead space volume given by

$$
V_{D}=0.1698 \dot{V}_{A}+0.1587
$$

However, some researchers have considered that equation (3) is only useful at rest or with low exercise. Mead (1960) showed that the respiratory frequency with moderate exercise may be determined more closely on the basis of optimal inspiratory pressure-time integral as a measure of the energy cost of breathing developed by the respiratory muscles. The equation obtained in (Mead, 1960) is

$$
f_{\text {Mead }}=\sqrt[3]{\frac{\gamma V_{A}}{4 \pi^{2} \lambda V_{D}}}
$$

Similarly, the optimization principle has also been applied to the prediction of airway caliber and dead space volume (Widdicombe and Nadel, 1963). Widdicombe found the following equation for the optimal respiratory frequency in moderate and severe exercise:

$$
f_{\text {Widdicombe }}=\sqrt{\frac{\gamma \dot{V_{A}}}{4 \lambda V_{D}}}
$$

These three alternatives have been used in the model (Fincham and Tehrani, 1983a) to evaluate differences in the respiratory variables at different levels of exercise. Note that the model (Khoo et al, 1991) considers a constant frequency of 15 breaths/minute and this variable is not included in the other models when it is one of the most important signals for the physiologists. 

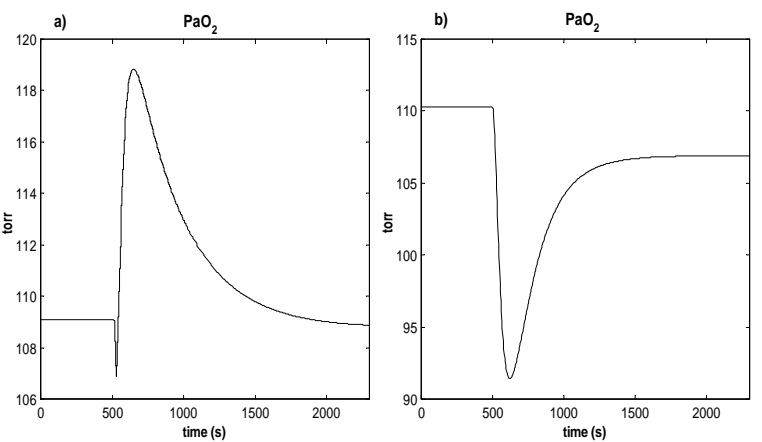

Fig. 6. Evolution of $\mathrm{PaO}_{2}$ in model a) (Fincham and Tehrani, 1983a) and b) (Khoo et al., 1991) when a step signal of $\dot{\mathrm{VCO}}_{2}$ is introduced at 500 seconds of the exercise simulation.

$\mathrm{V}_{\mathrm{E}}$ at different levels of exercise and $\mathrm{V}_{\mathrm{T}}$ versus $\mathrm{f}$ are shown in Fig. 7. Experimental data offered by the Hospital Clinic corresponding to a young healthy person is also included. Although the recorded data only give an idea, medical doctors suggest that $\mathrm{f}$ is usually between 12 and 15 breaths/minute at rest, and around 25 at maximum simulated exercise. Pressure values do not depend on the optimization frequency used as it happens with the transient response of all variables.
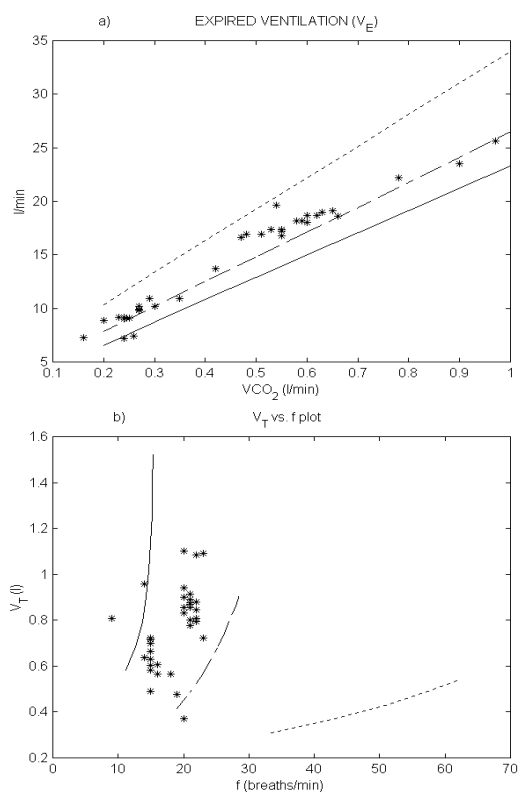

Fig. 7. a) $\mathrm{V}_{\mathrm{E}}$ as a function of $\mathrm{V}_{\mathrm{CO}_{2}}$, and b) $\mathrm{V}_{\mathrm{T}}$ versus $f$ at different levels of exercise. Different frequency equations are used: continuous (Otis et al, 1950), dashed (Mead, 1960), dotted (Widdicombe and Nadel, 1963). Experimental data from a healthy subject, '*', are also shown.

\subsection{Influence of time delay}

The presence of circulatory delays in gas transport permits to analyze their influence in the stability of the system. An abnormality of respiration called periodic breathing (PB) occurs in a number of different disease conditions. It is related with marginal stability in the control of ventilation and the most common type is Cheyne-Stokes breathing. (Guyton, 1986). The time delay that produces PB in the model (Fincham and Tehrani, 1983a) is $19 \mathrm{~s}$ when the normal value is $10 \mathrm{~s}$.

\subsection{Restrictive and obstructive pathologies}

An increase of the elastance, $\gamma$, and resistance, $\lambda$, are related to restrictive and obstructive lung diseases, respectively. Both mechanical parameters are included in respiratory frequency equations, (3), (5) and (6) in the model (Fincham and Tehrani, 1983a). An average expression between the equations (3) and (5) that depends on the $\mathrm{V} \mathrm{CO}_{2}$ is proposed to calculate the respiratory rate:

$$
f=k_{\text {Otis }} f_{\text {Otis }}+k_{\text {Mead }} f_{\text {Mead }}
$$

where

$$
k_{\text {Otis }}=-\frac{5}{8} \dot{V} \mathrm{CO}_{2}+\frac{8.2}{8} \quad k_{\text {Mead }}=\frac{5}{8} \mathrm{VCO}_{2}-\frac{0.2}{8}
$$

Both weights change linearly with the level of exercise and they sum always the unit. They are calculated to obtain an average of $90 \%$ and $10 \%$ at rest $\left(\mathrm{VCO}_{2}=0.2 \mathrm{l} / \mathrm{min}\right)$ and $40 \%$ and $60 \%$ with maximum simulated exercise $\left(\dot{\mathrm{V} \mathrm{CO}}_{2}=1 \mathrm{l} / \mathrm{min}\right)$ for $\mathrm{f}_{\mathrm{Otis}}$ and $\mathrm{f}_{\text {Mead }}$, respectively.

$\mathrm{V}_{\mathrm{E}}$ at different levels of exercise and $\mathrm{V}_{\mathrm{T}}$ versus $\mathrm{f}$ are shown in Fig. 8 for a healthy person and two loaded patients with five times the normal elastance and resistance. Equation (7) is considered in the model (Fincham and Tehrani, 1983a). Experimental data
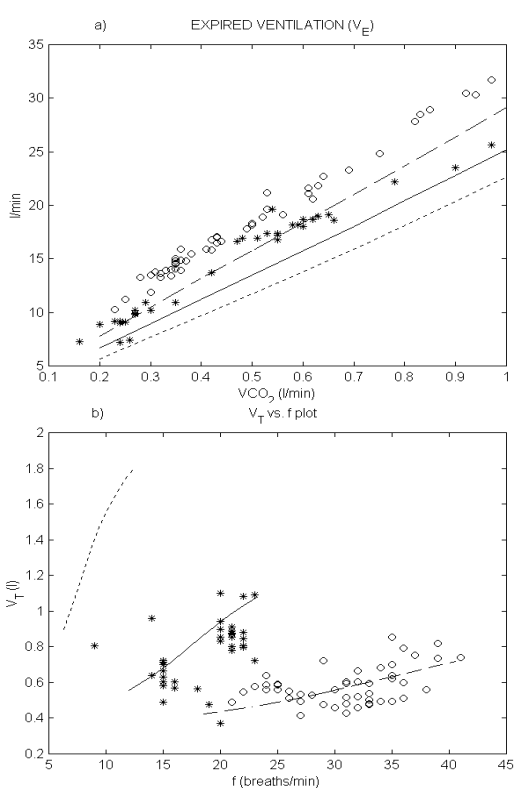

Fig. 8. a) $\mathrm{V}_{\mathrm{E}}$ as a function of $\mathrm{VCO}_{2}$, and b) $\mathrm{V}_{\mathrm{T}}$ versus $f$ at different levels of exercise in (Fincham and Tehrani, 1983a). Different line types are used to each respiratory case: normal subject (continuous), restrictive patient (dashed) and obstructive one (dotted). Experimental data from a healthy person, '*', and restrictive patient, 'o', are also shown. 
offered by the Hospital Clinic corresponding to a young healthy person and restrictive lung patient are also included. Pressure values and transient response are very similar between the three simulated subjects.

\section{CONCLUSIONS}

Mathematical models obtained from (Fincham and Tehrani, 1983a,b) and (Tehrani 1993) have permitted the implementation of a respiratory control system which is much more complete than the models (Poon, 1987) and (Khoo et al., 1982, 1991) and more useful for the medical doctors in some ventilatory situations. The most important difference is the presence of respiratory frequency that changes depending on the level of stimulus as it happens in fact. It is possible to obtain $V_{T}$ vs. f plots which are very interesting for the physiologists.

Comparing in general the steady-state responses, more differences have been obtained between (Fincham and Tehrani, 1983a) and (Khoo et al., 1982, 1991) than the first one with (Poon, 1987). This is because of the different control law and approach: The first ones depend on an optimal criterion whereas the last ones are regulated by means of proportional controller. In general for the three stimuli, the greatest variations are obtained in $\mathrm{V}_{\mathrm{E}}$, secondly $\mathrm{PaCO}_{2}$ and finally $\mathrm{PaO}_{2}$. On the other hand, the situation with more variations is moderate exercise. Although the model (Fincham and Tehrani, 1983a) does not keep homeostasis in exercise as well as (Poon, 1987), it is much better than Khoo's models. Otherwise, these last two models and the model (Fincham and Tehrani, 1983a) are most suitable to analyze hypoxia than (Poon, 1987). A practically systematic difference in $V_{E}$ among them is due to the absence of $\mathrm{PaO}_{2}$ feedback in the last model. Therefore, the ventilation and $\mathrm{PaCO}_{2}$ do not depend of the level of hypoxia $\left(\mathrm{P}_{\mathrm{I}} \mathrm{O}_{2}\right)$. However, the model (Fincham and Therani, 1983a) is not valid to evaluate levels of hypercapnia higher than $5 \%$ because of a non physiological decrease in $\mathrm{f}$.

Plots $V_{T}$ vs. f show a realistic physiological aspect: a higher increase in $f$ than $V_{T}$ with lower levels of exercise and hypercapnia. This effect tends to the opposite one when the stimulus increases. However, the values of $\mathrm{f}$ found with Otis equation are specially too small in medium-high level of simulated exercise tending to a maximum frequency with only moderate exercise. On the other hand, the optimization frequency proposed by (Widdicombe and Nadel, 1963 ) is clearly not suitable for these levels of exercise because its value is too high and $\mathrm{V}_{\mathrm{T}}$ is too small. An average between equations obtained from (Otis, 1950) and (Mead, 1960) is proposed to calculate the optimum frequency. The weights change linearly with the level of exercise so that the final $f$ tends slightly to $f_{\text {Mead }}$ with the increase of exercise. This approach is evaluated for healthy subjects, restrictive and obstructive patients.
Simulation results are compared with experimental data from two subjects for guidance only, but they adjust better physiological condition. Much more recorded data would be needed for a better adjustment of the weights. Therefore, the model is also suitable enough to evaluate respiratory mechanical dysfunctions.

Transient response of the model (Fincham and Tehrani, 1983) has overshoots in pressure variables and a settling time around two minutes in exercise as occurs in the human body response. However the response is too slow in the other stimuli. Finally, Periodic Breathing is produced with a threshold of 9 $\mathrm{s}$ in the extra-time delay of the gas circulation.

\section{REFERENCES}

Fincham W.F. and F.T. Tehrani (1983a) A mathematical model of the human respiratory system, Journal of Biomedical Engineering, vol. 5, April, pp. 125-133.

Fincham W. F. and F.T. Tehrani (1983b) On the regulation of cardiac output and cerebral blood flow. Journal of Biomedical Engineering, vol. 5, Jan., pp. 73-75.

Guyton, A.C. (1986) Textbook of Medical Physiology. Saunders, $7^{\text {th }}$ ed.

Khoo M. C., R.E. Kronauer, K.P. Strohl and A.S. Slutsky (1982) Factors inducing periodic breathing in humans: a general model. Journal of Applied Physiology, vol. 53, 3, pp. 644-659.

Khoo M. C., A. Gottschalk and A Pack (1991) Sleepinduced periodic breathing and apnea: a theoretical study. Journal of Applied Physiology, vol. 70, 5, pp. 2014-2024.

Mañanas, M.A., S. Romero and P. Caminal (2000) A comparative study of respiratory models in control of ventilation, Proc. Annual Int. Conf. IEEE-Eng. in Medicine and Biology Society, pp. 5491-5495.

Mead J. (1960) Control of respiratory frequency. Journal of Applied Physiology, vol. 15(3), pp. 325-336.

Otis A. B., W.O. Fenn and H. Rahn (1950) Mechanics of breathing in man. Journal of Applied Physiology, vol. 2, pp. 592-607.

Poon C-S. (1987) Ventilatory control in hypercapnia and exercise: optimization hypothesis. J. of Applied Physiology, vol. 62(6), pp. 2447-2459.

Poon, C-S (1995) Respiratory models and control, The Biomedical Engineering Handbook, CRC Press, Inc.

Tehrani F. T. (1993) Mathematical analysis and computer simulation of the respiratory system in the newborn infant. IEEE Transactions on Biomedical Engineering, vol. 40, no. 5, pp. 475481.

Widdicombe J.G. and J.A. Nadel (1963) Airway volume, airway resistance, and work of breathing: theory. Journal of Applied Physiology, vol 18(5), pp 863-868. 